\title{
Ionizing Cylindrical Shock Waves in a Rotating Homogeneous Non-ideal Gas
}

\author{
J. P. Vishwakarma*, Mahendra Singh \\ Dept. of Mathematics and Statistics, D.D.U. Gorakhpur University, Gorakhpur, 273009, India
}

\begin{abstract}
Similarity Solutions are obtained for one-dimensional adiabatic flow behind ionizing cylindrical shock wave propagating in a rotating non-ideal gas in presence of an azimuthal magnetic field. The electrical conductivity in the medium ahead of the shock is assumed to be negligible, which becomes infinitely large after passage of the shock. In order to obtain the similarity solutions, the initial density of the medium is assumed to be constant and the initial angular velocity to be obeying a power law and to be decreasing as the distance from the axis increases. The effects of an increase in the value of the index for variation of angular velocity of the ambient medium, in the value of the parameter of the non-idealness of the gas and in the strength of the ambient magnetic field on the shock propagation are investigated. It is observed that the non-idealness of the gas has decaying effect on the shock wave.
\end{abstract}

Keywords Ionizing Shock Wave, Azimuthal Magnetic Field, Non-Ideal Gas, Rotating Medium, Adiabatic Flow, Similarity Solutions

\section{Introduction}

The formulation of self-similar problems and examples describing the adiabatic motion of non-rotating gas models of stars, are considered by Sedov[1], Zel'dovich and Raizer[2], Lee and Chen[3] and Summers[4]. Rotation of the stars significantly affects the process taking place in their outer layers. Therefore, question connected with the explosions in the rotating gas atmospheres are of definite astrophysical interest. Chaturani[5] studied the propagation of cylindrical shock waves through a gas having solid body rotation and obtained the solutions by a similarity method adopted by Sakurai[6]. Nath, Ojha and Takhar[7] obtained the similarity solutions for the flow behind spherical shock waves propagating in a non-uniform rotating interplanetary atmosphere with increasing energy. Ganguly and Jana[8] studied a theoretical model of propagation of strong spherical shock waves in a self-gravitating atmosphere with radiation flux in presence of a magnetic field. They, also considered the medium behind the shock to be rotating, but neglected the rotation of the undisturbed medium. In all of the works, mentioned above, the medium is taken to be a gas satisfying the equation of state of a perfect gas. Because of high pressure and density that generally occur behind a shock wave, produced by an explosion, the assumption that the gas is ideal is no more valid. The popular alternative to

* Corresponding author:

jpv_univgkp@yahoo.com (J. P. Vishwakarma)

Published online at http://journal.sapub.org/mechanics

Copyright (C) 2012 Scientific \& Academic Publishing. All Rights Reserved the ideal gas is a simplified van der Waals model. Roberts and $\mathrm{Wu}[9,10]$ adopted this model to discuss the shock wave theory of Sonoluminescence. Vishwakarma et al.[11] too adopted this as their model of a non-ideal gas to obtain the self-similar solutions for the flow behind a magnetogasdynamic cylindrical shock wave propagating in a rotating gas in presence of an azimuthal magneting field. They have taken the electrical conductivity of the initial medium and the medium behind the shock to be infinite. But, in many practical cases the medium may be of low conductivity which becomes highly conducting due to passage of a strong shock. Such a shock wave is called a gas-ionizing shock or, simply ionizing shock. The propagation of a ionizing shock has been studied by Greenspan[12,] Greifinger and Cole[13], Christer[14], Rangarao andRamana[15] and Singh[16] in a non-rotating perfect gas. In the present work, we have extended the work of Vishwakarma et al.[11] by investigating the propagation of gas-ionizing shock in a non-ideal rotating gas in place of magnetogasdynamic shock. In order to obtain similarity solutions, the initial density of the medium is assumed to be constant and the initial angular velocity of rotation to be obeying a power law and to be decreasing as the distance from the axis increases. It is expected that such an angular velocity may occur in the atmospheres of rotating stars.

Effects of a change in the strength of ambient magnetic field, in the non-idealness of the gas and in the index of variation of angular velocity of the ambient medium (or index of variation of ambient magnetic field) are investigated. A comparison is also made between the results of the present work and those of the corresponding magnetogasdynamic 
shocks.

\section{Basic Equations and Boundary Conditions}

The fundamental equations governing the unsteady adiabatic cylindrically symmetric motion of a non-ideal and perfectly conducting gas, which is rotating about the axis of symmetry and in which an azimuthal magnetic field is permeated and heat conduction and viscous stress are negligible (c.f. Whitham[17], Chaturani[5]), are:

$$
\begin{gathered}
\frac{\partial \rho}{\partial \mathrm{t}}+\mathrm{u} \frac{\partial \rho}{\partial \mathrm{r}}+\rho \frac{\partial \mathrm{u}}{\partial \mathrm{r}}+\frac{\rho \mathrm{u}}{\mathrm{r}}=0 \\
\frac{\partial \mathrm{u}}{\partial \mathrm{t}}+\mathrm{u} \frac{\partial \mathrm{u}}{\partial \mathrm{r}}+\frac{1}{\rho}\left(\frac{\partial \mathrm{p}}{\partial \mathrm{r}}+\mu \mathrm{h} \frac{\partial \mathrm{h}}{\partial \mathrm{r}}+\frac{\mu \mathrm{h}^{2}}{\mathrm{r}}\right)-\frac{\mathrm{v}^{2}}{\mathrm{r}}=0 \\
\frac{\partial \mathrm{h}}{\partial \mathrm{t}}+\mathrm{u} \frac{\partial \mathrm{h}}{\partial \mathrm{r}}+\mathrm{h} \frac{\partial \mathrm{u}}{\partial \mathrm{r}}=0 \\
\frac{\partial \mathrm{v}}{\partial \mathrm{t}}+\mathrm{u} \frac{\partial \mathrm{v}}{\partial \mathrm{r}}+\frac{\mathrm{uv}}{\mathrm{r}}=0 \\
\frac{\mathrm{de}}{\mathrm{dt}}+\mathrm{p} \frac{\mathrm{d}}{\mathrm{dt}}\left(\frac{1}{\rho}\right)=0
\end{gathered}
$$

where $\frac{\mathrm{d}}{\mathrm{dt}}=\frac{\partial}{\partial \mathrm{t}}+\mathrm{u} \frac{\partial}{\partial \mathrm{r}}$

and $\rho, \mathrm{p}, \mathrm{h}$ are the density, pressure and azimuthal magnetic field, respectively, $\mathrm{u}$ and $\mathrm{v}$ are the radial and azimuthal components of the fluid velocity, $\mu$ is the magnetic permeability, $r$ and $t$ are the distance and time, and $\mathrm{e}$ is the internal energy per unit mass. Also, we have

$$
\mathrm{v}=\mathrm{Ar} \text {, }
$$

where $\mathrm{A}$ is the angular velocity of the medium at the radial distance $r$ from the axis of the symmetry.

In most of the cases the propagation of shock waves arises in extreme conditions under which the assumption that the gas is ideal is not a sufficiently accurate description. To discover how deviations from the ideal gas can affect the solutions, we adopt a simple model. We assume that the gas obeys a simplified van der Waals equation of state of the form (Roberts and $\mathrm{Wu}[9],[10]$ )

$$
\begin{gathered}
\mathrm{p}=\frac{\Gamma \rho \mathrm{T}}{(1-\mathrm{b} \rho)}, \\
\mathrm{e}=\mathrm{C}_{\mathrm{v}} \mathrm{T}=\frac{\mathrm{p}(1-\mathrm{b} \rho)}{\rho(\gamma-1)},
\end{gathered}
$$

where $\Gamma$ is the gas constant, $\mathrm{C}_{\mathrm{v}}=\frac{\Gamma}{\gamma-1}$ is the specific heat at constant volume and $\gamma$ is the ratio of specific heats. The constant $b$ is the 'van der Waals excluded volume'; it places a limit, $\rho_{\max }=\frac{1}{b}$, on the density of the gas.

We assume that a cylindrical shock is propagating outwards from the axis of symmetry in a rotating non-ideal gas with constant initial density and negligible electrical con- ductivity in presence of an azimuthal magnetic field. Due to passage of the shock, the gas is highly ionized and its electrical conductivity becomes infinitely large. The conditions across such a gas-ionizing shock are (c.f. Singh and Srivastava[18] and Vishwakarma and Pandey[19])

$$
\begin{gathered}
\mathrm{u}_{2}=(1-\beta) \mathrm{V}, \\
\rho_{2}=\frac{\rho_{1}}{\beta}, \\
\mathrm{h}_{1}=\mathrm{h}_{2}, \\
\mathrm{p}_{2}=\left[\frac{1}{\gamma \mathrm{M}^{2}}+(1-\beta)\right] \rho_{1} \mathrm{~V}^{2}, \\
\mathrm{v}_{2}=\mathrm{v}_{1},
\end{gathered}
$$

where $\beta=\frac{1}{2(\gamma+1)}[\{(1-\bar{b})(\gamma-1)\}+$

$$
\left.\sqrt{(\gamma-1)\left\{(1-\overline{\mathrm{b}})^{2}(\gamma-1)+4 \overline{\mathrm{b}}(\gamma+1)\right\}}\right]
$$

and $\bar{b}=b \rho_{1}$ is the parameter of non-idealness of the gas. Here $\mathrm{V}$ is the shock velocity, $\mathrm{M}$ is the shock-Mach number referred to frozen speed of sound $\left(\frac{\gamma \mathrm{p}_{1}}{\rho_{1}}\right)^{1 / 2}$, and $\mathrm{M}_{\mathrm{A}}$ is the Alfven-Mach number. Quantities with suffices ' 1 ' and ' 2 ' correspond to their values just ahead and just behind the shock, respectively.

The shock-Mach number $M_{e}$ referred to the speed of sound in non-ideal gas $\left[\frac{\gamma p_{1}}{\rho_{1}(1-\bar{b})}\right]^{1 / 2}$ and the Alfven-Mach number $\mathrm{M}_{\mathrm{A}}$ are given by

$$
\begin{aligned}
& \text { And } M_{A}=\frac{V}{\left(\mu h_{1}^{2} / \rho_{1}\right)^{1 / 2}}, \\
& \text { where } M=\frac{V}{\left(\gamma p_{1} / \rho_{1}\right)^{1 / 2}} .
\end{aligned}
$$

Ahead of the shock the azimuthal magnetic field varies as

$$
\mathrm{h}_{1}=\mathrm{h}_{0} \mathrm{R}^{\alpha} \text {, }
$$

where $h_{0}$ and $\alpha$ are constants, and $R$ is the shock radius.

In order to obtain the similarity solutions it is assumed that the initial angular velocity $A_{1}$ varies as

$$
\mathrm{A}_{1}=\mathrm{A}_{0} \mathrm{R}^{\mathrm{d}}
$$

where $\mathrm{A}_{0}$ and $\mathrm{d}$ are constants.

The momentum equation (2.2) in the undisturbed state of the gas, gives

$$
\mathrm{p}_{1}=\rho_{1} \mathrm{~A}_{0}^{2} \frac{\mathrm{R}^{2(1+\mathrm{d})}}{2(1+\mathrm{d})}-(1+\alpha) \mu \mathrm{h}_{0}^{2} \frac{\mathrm{R}^{2 \alpha}}{2 \alpha}+\text { constant. }
$$

The total energy of the flow-field behind the shock is not constant, but assumed to be time dependent and varying as (Rogers[20], Freeman[21], Director and Dabora[22])

$$
\mathrm{E}=\mathrm{E}_{0} \mathrm{t}^{\omega}, \quad \omega \geq 0,
$$

where $\mathrm{E}_{0}$ and $\omega$ are constants. The positive value of $\omega$ correspond to the class in which the total energy increases 
with time. This increase can be achieved by the pressure exerted on the fluid by an expanding surface (a contact surface or a piston). This surface may be, physically, the surface of the stellar corona or the condensed explosives or the diaphragm containing a very high-pressure driver gas. By sudden expansion of the stellar corona or the detonation products or the driver gas into the ambient gas, a shock wave is produced in the ambient gas. The shocked gas is separated from this expanding surface which is a contact discontinuity. This contact surface acts as a 'piston' for the shock wave. Thus the flow is headed by a shock front and has an expanding surface as an inner boundary. The situation very much of the same kind may prevail in the formation of cylindrical spark channel from exploding wires. In addition, in the usual cases of spark break down, time dependent energy input is a more realistic assumption than instantaneous energy input (Freeman and Cragges[23]).

\section{Similarity Solutions}

Zel'dovich and Raizer[2] shown that the gasdynamic equations admit similarity transformations, that there are possible different flows similar to each other which are derivable from each other by changing the basic scales of length, time, and density. The motion itself may be described by the most general functions of the two variables $r$ and $t$, $\rho(r, t), p(r, t), u(r, t), v(r, t)$ and $h(r, t)$. These functions also contain the parameters entering the initial and boundary conditions of the problem (and specific heat ratio $\gamma$ ).

However, there exist motions whose distinguishing property is the similarity in the motion itself. These motions are called self-similar[1, 2]. The distribution as a function of position of any of the flow variables, such as the pressure $\mathrm{p}$, evolves with time in a self-similar motion in such a manner that only the scale of the pressure $\Pi(t)$ and the length scale $\mathrm{R}(\mathrm{t})$ of the region included in the motion change, but the shape of the pressure distribution remains unaltered. The $\mathrm{p}(\mathrm{r})$ curves corresponding to different time $t$ can be made the same by either expanding or contracting the $\Pi$ and the R scales. The function $\mathrm{p}(\mathrm{r}, \mathrm{t})$ can be written in the form

$$
\mathrm{p}(\mathrm{r}, \mathrm{t})=\Pi(\mathrm{t}) \mathrm{P}\left(\frac{\mathrm{r}}{\mathrm{R}}\right),
$$

where the dimensional scales $\Pi$ and $\mathrm{R}$ depend on time in some manner, and the dimensionless ratio $\frac{\mathrm{p}}{\Pi}=\mathrm{P}\left(\frac{\mathrm{r}}{\mathrm{R}}\right)$ is a "universal" (in the sense that it is independent of time) function of the new dimensionless coordinate $x=\frac{r}{R}$. Multiplying the variables $\mathrm{P}\left(\frac{\mathrm{r}}{\mathrm{R}}\right)$ and $\mathrm{x}$ by the scale functions $\Pi(\mathrm{t})$ and $\mathrm{R}(\mathrm{t})$, we can obtain from the universal function $\mathrm{P}(\mathrm{x})$ the true pressure distribution curve $p(r)$ as a function of position for any time t. The other flow variables, density, velocity and magnetic field are expressed similarly.

For self-similar motions the system of partial differential equations (2.1)-(2.5) reduces to a system of ordinary differential equations in new unknown functions of the similarity variable $\mathrm{x}=\frac{\mathrm{r}}{\mathrm{R}}$. Let us derive these equations. To do this we represent the solution of the partial differential equations (2.1)-(2.5) in terms of products of scale functions and the new unknown functions of the similarity variable $\mathrm{x}$,

$$
\mathrm{x}=\frac{\mathrm{r}}{\mathrm{R}}, \mathrm{R}=\mathrm{R}(\mathrm{t}) \text {. }
$$

The pressure, density, velocity, magnetic field, and length scales are not all independent of each other. If we have choose $\mathrm{R}$ and $\rho_{1}$ as the basic scales, then the quantity $\mathrm{V}=\mathrm{F}$ can serve as the velocity scale, $\rho_{1} \mathrm{~V}^{2}$ as the pressure scale, and $\sqrt{\left(\rho_{1} / \mu\right)} \mathrm{V}$ as the magnetic field scale. This does not limit generality of the solution, as the scale is only defined to within a numerical coefficient which can always be included in the new unknown function. We seek a solution of the form

$$
\begin{gathered}
\mathrm{u}=\mathrm{VU}(\mathrm{x}), \\
\rho=\rho_{1} \Omega(\mathrm{x}), \\
\mathrm{p}=\rho_{1} \mathrm{~V}^{2} \mathrm{P}(\mathrm{x}), \\
\mathrm{v}=\mathrm{VK}(\mathrm{x}), \\
\sqrt{\mu} \mathrm{h}=\left(\rho_{1}\right)^{1 / 2} \mathrm{VH}(\mathrm{x}) .
\end{gathered}
$$

whereU, $\Omega, \mathrm{P}, \mathrm{K}$ and $\mathrm{H}$ are new dimensionless functions of the similarity variable $\mathrm{x}$, in terms of which the differential equations are to be formulated. The shock front is represented by $\mathrm{x}=1$.

The shock conditions (2.9) to (2.13) are transformed into

$$
\begin{gathered}
\mathrm{U}(1)=1-\beta, \\
\Omega(1)=\frac{1}{\beta}, \\
\mathrm{H}(1)=\frac{1}{\mathrm{M}_{\mathrm{A}}}, \\
\mathrm{P}(1)=\frac{\mathrm{p}_{2}}{\rho_{1} \mathrm{~V}^{2}}\left[\frac{1}{\gamma \mathrm{M}^{2}}+(1-\beta)\right], \\
\mathrm{K}(1)=\left[\frac{2 \alpha}{\gamma \mathrm{M}^{2}}+\frac{1+\alpha}{\mathrm{M}_{\mathrm{A}}^{2}}\right]^{1 / 2},
\end{gathered}
$$

where $1+d=\alpha$.

In the derivation of the condition (3.11) it was necessary to use the relation (3.12), otherwise (3.11) would contain $\mathrm{R}(\mathrm{t})$ and the solution would not be self-similar.

The total energy behind the shock is given by

$$
\mathrm{E}=2 \pi \int_{\mathrm{r}_{\mathrm{p}}}^{\mathrm{R}}\left\{\frac{1}{2} \rho\left(\mathrm{v}^{2}+\mathrm{u}^{2}\right)+\frac{\mathrm{p}(1-\mathrm{b} \rho)}{\gamma-1}+\frac{\mu \mathrm{h}^{2}}{2}\right\} \mathrm{rdr}=\mathrm{E}_{0} \mathrm{t}^{\omega},
$$

where $r_{p}$ is the radius of inner expanding surface. Applying the similarity transformations (3.2) to (3.6) to the relation (3.13), we find that the motion of the shock front is given by the equation

$$
\mathrm{R}^{2} \mathrm{~V}^{2}=\frac{\mathrm{E}_{0} \mathrm{t}^{\omega}}{2 \pi \rho_{1} \mathrm{~J}}
$$


where $J=\int_{x_{\mathrm{p}}}^{1}\left[\frac{1}{2} \Omega\left(\mathrm{U}^{2}+\mathrm{K}^{2}\right)+\frac{\mathrm{P}(1-\overline{\mathrm{b}} \Omega)}{(\gamma-1)}+\frac{\mathrm{H}^{2}}{2}\right] \mathrm{xdx}$

in which $\mathbf{x}_{\mathrm{p}}$ is the value of $\mathrm{x}$ at the inner expanding surface.

Equation (3.14) can be written as

$$
\mathrm{R} \frac{\mathrm{dR}}{\mathrm{dt}}=\left(\frac{\mathrm{E}_{0}}{2 \pi \rho_{1} \mathrm{~J}}\right)^{1 / 2} \mathrm{t}^{\mathrm{\omega} / 2}
$$

which on integration gives

$$
\mathrm{R}=\left(\frac{8 \mathrm{E}_{0}}{\pi \rho_{1} \mathrm{~J}}\right)^{1 / 4} \frac{1}{\sqrt{\omega+2}} \mathrm{t}^{(\omega+2) / 4} .
$$

From (3.17) we get the shock velocity

$$
\mathrm{V}=\frac{\mathrm{dR}}{\mathrm{dt}}=\frac{(\omega+2) \mathrm{R}}{4 \mathrm{t}}=\left(\frac{8 \mathrm{E}_{0}}{\pi \rho_{1} \mathrm{~J}}\right)^{1 / \omega+2} \frac{(\omega+2)^{\omega / \omega+2}}{4} \mathrm{R}^{\frac{\omega-2}{\omega+2}}
$$

Since $\mathrm{M}$ and $\mathrm{M}_{\mathrm{A}}$ are constants for similarity solutions, we have

$$
\alpha=\frac{\omega-2}{\omega+2} .
$$

Now, the following two cases arise

Case 1: $\alpha=0$

In this case, we have

$$
\mathrm{d}+1=0
$$

and the shock velocity is constant. It follows that $\mathrm{p}_{1}=$ constant,

$$
\text { and } \mathrm{M}^{2}=\frac{\mu \mathrm{h}_{0}^{2} \mathrm{M}_{\mathrm{A}}^{2}}{\gamma \mathrm{p}_{1}} \text {. }
$$

Case 2: $\alpha \neq 0$.

In this case, the constant in the right hand side of (2.20) must be zero, and the shock velocity is variable and so

$$
\mathrm{M}^{2}=\frac{2 \alpha \mu \mathrm{h}_{0}^{2} \mathrm{M}_{\mathrm{A}}^{2}}{\gamma\left[\rho_{1} \mathrm{~A}_{0}^{2}-(1+\alpha) \mu \mathrm{h}_{0}^{2}\right]} .
$$

Now, we have the following relations valid in both the case:

$$
\begin{aligned}
& \frac{\mu \mathrm{h}_{0}^{2} \mathrm{M}_{\mathrm{A}}^{2}}{\rho_{1}}=\left[\left(\frac{8 \mathrm{E}_{0}}{\pi \rho_{1} \mathrm{~J}}\right)^{1 / \omega+2} \frac{(\omega+2)^{\omega / \omega+2}}{4}\right]^{2}, \\
& \text { And } \frac{\mathrm{A}_{0}^{2} \rho_{1}}{\mu \mathrm{h}_{0}^{2}}=\frac{2 \alpha \mathrm{M}_{\mathrm{A}}^{2}}{\gamma \mathrm{M}^{2}}+(1+\alpha), \quad 0<\mathrm{M}_{\mathrm{A}}<\infty \text {. }
\end{aligned}
$$

To obtain the solution in a convenient form, we introduce the following transformations:

$$
\begin{aligned}
\mathrm{g} & =\frac{\rho}{\rho_{2}}, \\
\mathrm{y} & =\frac{\mathrm{p}}{\mathrm{p}_{2}}, \\
\mathrm{~W} & =\frac{\mathrm{u}}{\mathrm{V}}, \\
\mathrm{z} & =\frac{\mathrm{v}}{\mathrm{V}},
\end{aligned}
$$

$$
\mathrm{s}=\frac{\mathrm{h}}{\mathrm{h}_{2}} .
$$

Using the transformations (3.26) to (3.30), the equations of the motion (2.1) to (2.5) take the form

$$
\begin{gathered}
(W-x) \frac{d g}{d x}+g \frac{d W}{d x}+\frac{g W}{x}=0 \\
(W-x) \frac{d W}{d x}+\frac{\beta F}{g} \frac{d y}{d x}+\frac{\beta s}{g M_{A}^{2}} \frac{d s}{d x}+\frac{\beta s^{2}}{g x M_{A}^{2}}+\alpha W-\frac{z^{2}}{x}=0 \\
(W-x) \frac{d s}{d x}+s \frac{d W}{d x}+\alpha s=0 \\
(W-x) \frac{d z}{d x}+\frac{W z}{x}+\alpha z=0 \\
2 \alpha y+(W-x) \frac{d y}{d x}-\frac{\gamma y \beta(W-x)}{g(\beta-\bar{b} g)} \frac{d g}{d x}=0 \\
\text { where } F=\frac{1}{\gamma M^{2}}+(1-\beta)
\end{gathered}
$$

In terms of the dimensionless variables $\mathrm{x}, \mathrm{W}, \mathrm{y}, \mathrm{g}, \mathrm{s}$, and $\mathrm{z}$, The shock conditions take the form

$$
\begin{gathered}
\mathrm{x}=1, \\
\mathrm{~W}=1-\beta, \\
\mathrm{g}=1, \\
\mathrm{~s}=1, \\
\mathrm{y}=1, \\
\mathrm{z}=\left[\frac{(1+\alpha)}{\mathrm{M}_{\mathrm{A}}^{2}}+\frac{2 \alpha}{\gamma \mathrm{M}^{2}}\right]^{1 / 2} .
\end{gathered}
$$

Because of the dependence of the equations (3.31) to (3.35) and (3.38) on $\bar{b}$, similarity solution exists only when $\bar{b}$ is constant, i.e. only when the initial density $\rho_{1}$ is constant. The problem with the flow of a non-ideal gas is different from that of the perfect gas problem. In the latter case, similarity solution exists for initial density varying as some power of distance (Rogers[20], Rosenau[24]), but it is not true for the problem with the flow of a non-ideal gas.

In addition to the shock conditions(3.37) to (3.42), the condition to be satisfied at the inner boundary surface is that the velocity of the fluid is equal to the velocity of inner boundary itself. This kinematic condition, from equations (3.2) and (3.28), can be written as

$$
\mathrm{W}\left(\mathrm{x}_{\mathrm{p}}\right)=\mathrm{x}_{\mathrm{p}} .
$$

From equations (3.31) to (3.35), we have

$$
\begin{aligned}
\mathrm{Bx} \frac{\mathrm{dW}}{\mathrm{dx}} & =2 \alpha \beta \mathrm{xyF}+\frac{\gamma \mathrm{y} \beta^{2} \mathrm{FW}}{(\beta-\overline{\mathrm{b} g})} \\
& -(\mathrm{W}-\mathrm{x})\left[\frac{\mathrm{s}^{2} \beta}{\mathrm{M}_{\mathrm{A}}^{2}}-\mathrm{z}^{2} \mathrm{~g}+\alpha \mathrm{gWx}+\frac{\alpha \mathrm{s}^{2} \beta \mathrm{x}}{\mathrm{M}_{\mathrm{A}}^{2}}\right],
\end{aligned}
$$




$$
\begin{aligned}
& \mathrm{Bx}(\mathrm{W}-\mathrm{x}) \frac{\mathrm{ds}}{\mathrm{dx}}= \\
& -\mathrm{s}\left[\alpha \mathrm{x}\left\{\mathrm{g}(\mathrm{W}-\mathrm{x})^{2}-\frac{\mathrm{s}^{2} \beta}{\mathrm{M}_{\mathrm{A}}^{2}}\right\}\right. \\
& +(1-\alpha x) \frac{\gamma y \beta^{2} F W}{(\beta-\bar{b} g)}+2 \alpha \beta x y F \\
& \left.-(\mathrm{W}-\mathrm{x})\left\{\frac{\mathrm{s}^{2} \beta}{\mathrm{M}_{\mathrm{A}}^{2}}-\mathrm{z}^{2} \mathrm{~g}+\alpha \mathrm{xgW}\right\}+\frac{\alpha \mathrm{s}^{2} \beta \mathrm{x}}{\mathrm{M}_{\mathrm{A}}^{2}}\right], \\
& \mathrm{Bx}(\mathrm{W}-\mathrm{x}) \frac{\mathrm{dg}}{\mathrm{dx}}= \\
& g\left[-2 \alpha \beta x y F+(W-x)\left\{\frac{s^{2} \beta}{M_{A}^{2}}-z^{2} g+\alpha g W x\right\}\right. \\
& -\frac{\alpha \beta s^{2} x}{M_{A}^{2}}-W\left\{(W-x)^{2} g-\frac{s^{2} \beta}{M_{A}^{2}}\right\}, \\
& B x(W-x) \frac{d y}{d x} \\
& =\frac{\gamma y \beta}{(\beta-\bar{b} g)}\left[(W-x)\left\{\frac{s^{2} \beta}{M_{A}^{2}}-z^{2} g+\alpha g W x\right\}\right. \\
& \left.-\frac{\alpha \mathrm{s}^{2} \beta \mathrm{x}}{\mathrm{M}_{\mathrm{A}}^{2}}-\mathrm{W}\left\{(\mathrm{W}-\mathrm{x})^{2} \mathrm{~g}-\frac{\beta \mathrm{s}^{2}}{\mathrm{M}_{\mathrm{A}}^{2}}\right\}\right] \\
& -2 \alpha x y\left[(W-x)^{2} g-\frac{s^{2} \beta}{M_{A}^{2}}\right], \\
& x(W-x) \frac{d z}{d x}=-z(\alpha x+W),
\end{aligned}
$$

Now, the equations (3.44) to (3.48) may be integrated, numerically, with the boundary conditions (3.37) to (3.42) and the appropriate values of the constant parameters $\gamma, \alpha$, $\bar{b}, M$ and $M_{A}$, to obtain $W, g, s, y$ and $z$.

\section{Results and Discussion}

Similarity considerations led to the following relations among the constants $\alpha$, $d$ and $\omega$ :

$$
\begin{gathered}
1+d=\alpha, \\
\alpha=\frac{\omega-2}{\omega+2} .
\end{gathered}
$$

Thus, the shock velocity varies as the ambient azimuthal velocity of the medium or as the ambient magnetic field.

Then the following two cases may exist,

(i) The constant velocity shock $(\alpha=0)$;

(ii) The decreasing velocity shock $(\alpha<0)$.

Therefore, for the purpose of numerical calculations, we choose $\alpha=0,-0.5$ which correspond, respectively, to the following two sets of values of the constants:

(i) $\alpha=0, \omega=2, d=-1$, and

(ii) $\alpha=-\frac{1}{2}, \omega=\frac{2}{3}, \mathrm{~d}=-\frac{3}{2}$.

The solution of the differential equations (3.44) to (3.48) with boundary conditions (3.37) to (3.42) depends on five constant parameters $\gamma, M, M_{A}, \bar{b}$ and $\alpha$. Numerical integration of these differential equations is performed to obtain the reduced variables $\mathrm{W}, \mathrm{z}$, g, $\mathrm{y}, \mathrm{s}$, starting from the shock surface to the inner expanding surface for $\gamma=5 / 3 ; \mathrm{M}=10 ; \mathrm{M}_{\mathrm{A}}^{-2}=0.02,0.1 ; \overline{\mathrm{b}}=0,0.05,0.1 ; \alpha=0$, -0.5 (Rosenau and Frankenthal[25], Rosenau[24], Vishwakarma and Yadav[26], Vishwakarma and Singh[27]). For a fully ionized gas $\gamma=5 / 3$, and therefore it is applicable to stellar medium. Rosenau and Frankenthal[25] have shown that the effects of magnetic field on the flow-field behind the shock are significant when $\mathrm{M}_{\mathrm{A}}^{-2} \geq 0.01$. Therefore the above values of $\mathrm{M}_{\mathrm{A}}^{-2}$ are taken for calculations in the present problem. The value $\overline{\mathrm{b}}=0$ corresponds to the perfect gas case. The results are shown in figures 1-5. Values of $\mathbf{x}_{\mathrm{p}}$ (the reduced position of the inner expanding surface) and the density ratio across the shock front $\beta=\rho_{1} / \rho_{2}$ are shown in tables 1 and 2 for different cases.

Table 1. Position of inner expanding surface $x_{p}$ for $\gamma=5 / 3, M=10$ and various values of $\mathrm{M}_{\mathrm{A}}^{-2}, \mathrm{~b}$, and $\alpha$

\begin{tabular}{|c|c|c|c|c|c|}
\hline & & & & \multicolumn{2}{|c|}{$\mathrm{x}_{\mathrm{p}}$} \\
$\mathrm{M}_{\mathrm{A}}^{-2}$ & $\overline{\mathrm{b}}$ & $\alpha$ & $\omega$ & \multicolumn{2}{|c|}{} \\
\cline { 3 - 6 } & & & & $\begin{array}{c}\text { magnetogasdynamic } \\
\text { Shock (Vishwa- } \\
\text { karma et al.[11]) }\end{array}$ & $\begin{array}{c}\text { Gas-ionizing } \\
\text { Shock (Our } \\
\text { Case) }\end{array}$ \\
\hline 0.02 & 0.0 & -0.5 & & 0.656 & 0.762851 \\
0.02 & 0.05 & -0.5 & $2 / 3$ & 0.628 & 0.747620 \\
0.02 & 0.1 & -0.5 & & 0.612 & 0.740120 \\
\hline 0.1 & 0.0 & -0.5 & & 0.473 & 0.731528 \\
0.1 & 0.05 & -0.5 & $2 / 3$ & 0.446 & 0.711262 \\
0.1 & 0.1 & -0.5 & & 0.456 & 0.699147 \\
\hline 0.02 & 0.0 & 0.0 & & 0.820 & 0.865428 \\
0.02 & 0.05 & 0.0 & 2 & 0.806 & 0.847192 \\
0.02 & 0.1 & 0.0 & & 0.788 & 0.832530 \\
\hline 0.1 & 0.0 & 0.0 & & 0.727 & 0.852846 \\
0.1 & 0.05 & 0.0 & 2 & 0.714 & 0.834035 \\
0.1 & 0.1 & 0.0 & & 0.711 & 0.819464 \\
\hline
\end{tabular}

Table 2. Density ratio $\beta$ across the shock front for $\bar{b}=0,0.05,0.1$, $\mathrm{M}=10$ and $\gamma=5 / 3$

\begin{tabular}{|c|c|}
\hline & $\beta=\rho_{1} / \rho_{2}$ \\
& \\
\hline & \\
0.0 & 0.16667 \\
0.05 & 0.20000 \\
0.1 & 0.22430394 \\
\hline
\end{tabular}





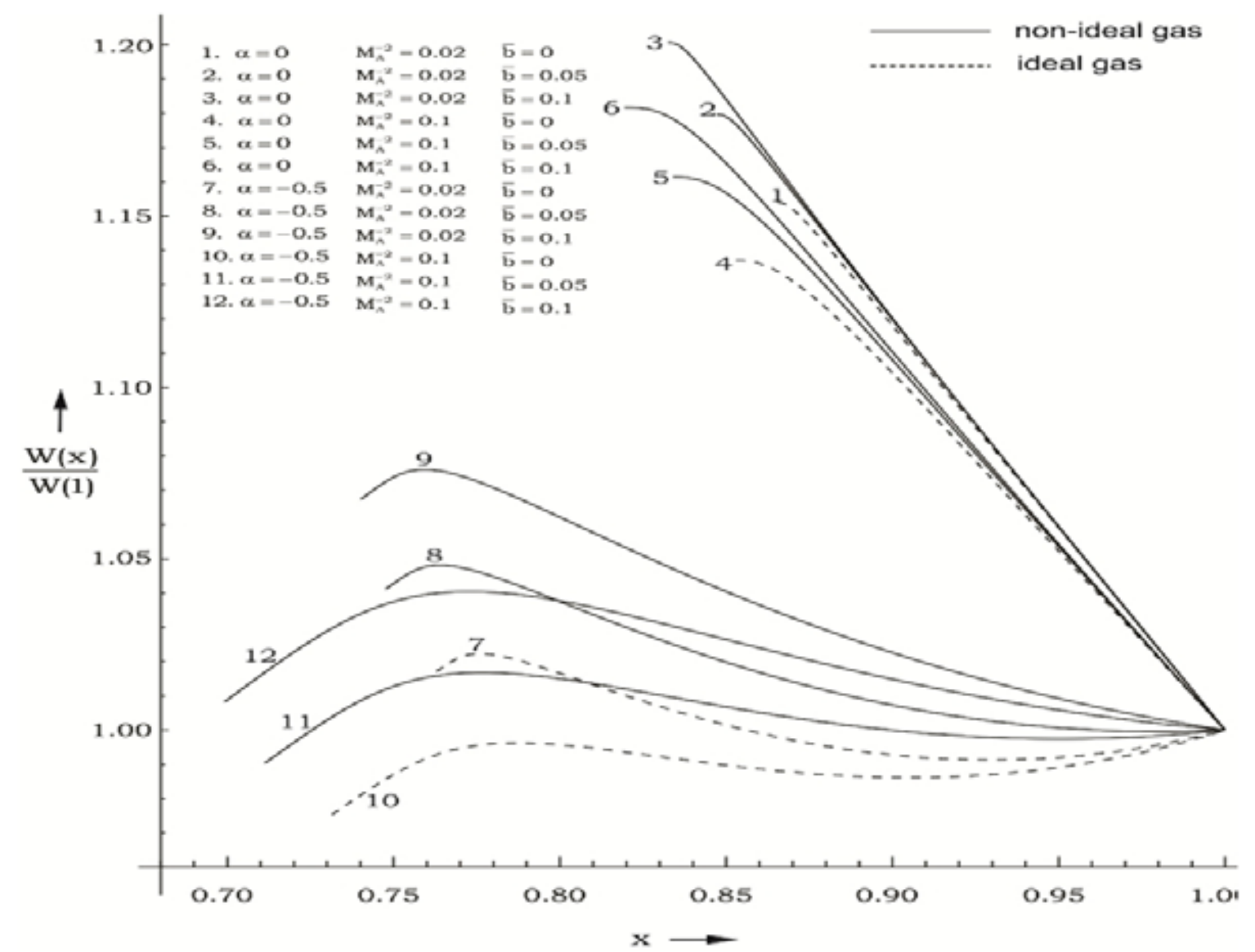

Figure 1. Variation of the reduced radial velocity in the flow-field behind the shock front

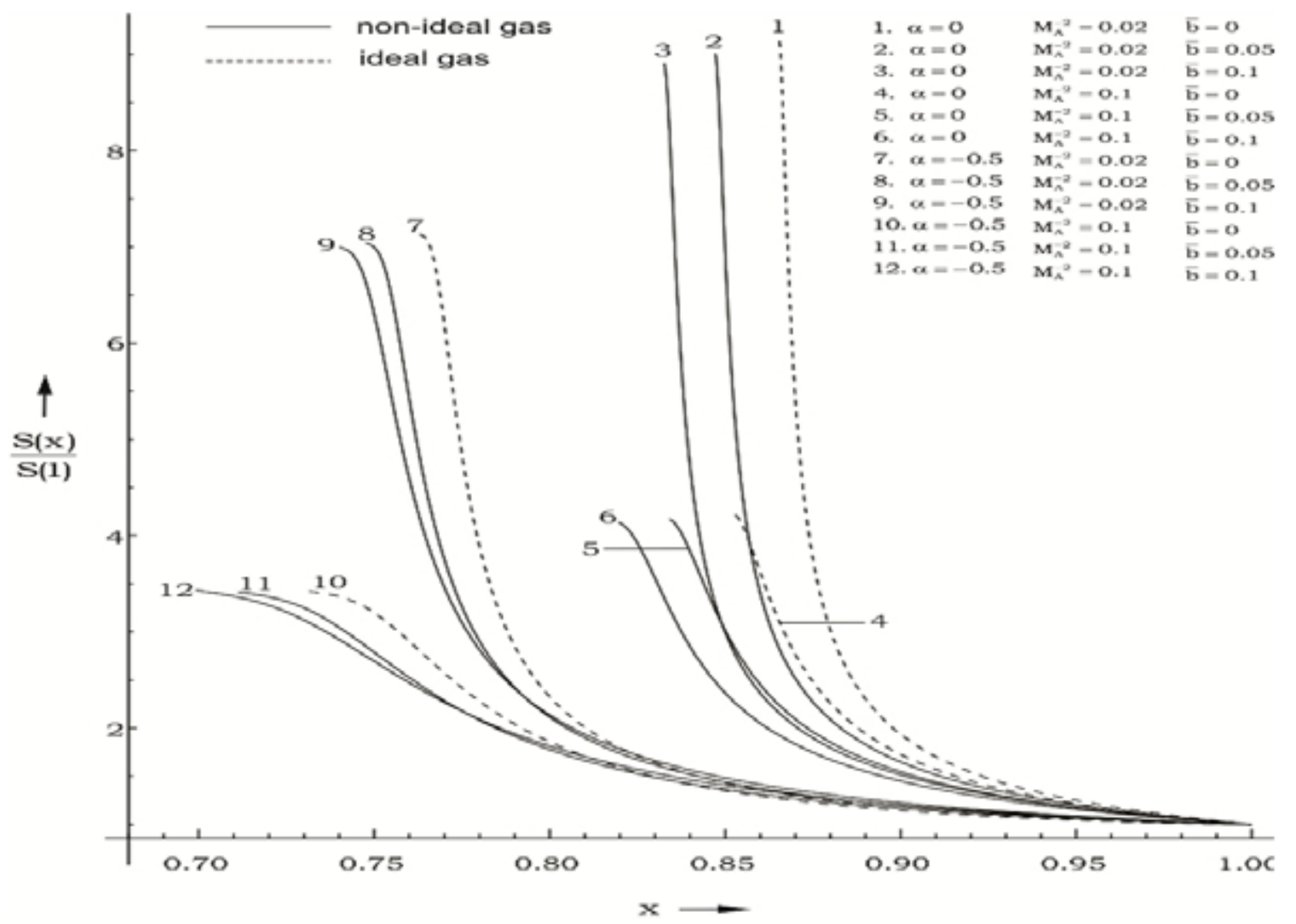

Figure 2. Variation of the reduced azimuthal magnetic field in the flow-field behind the shock front 


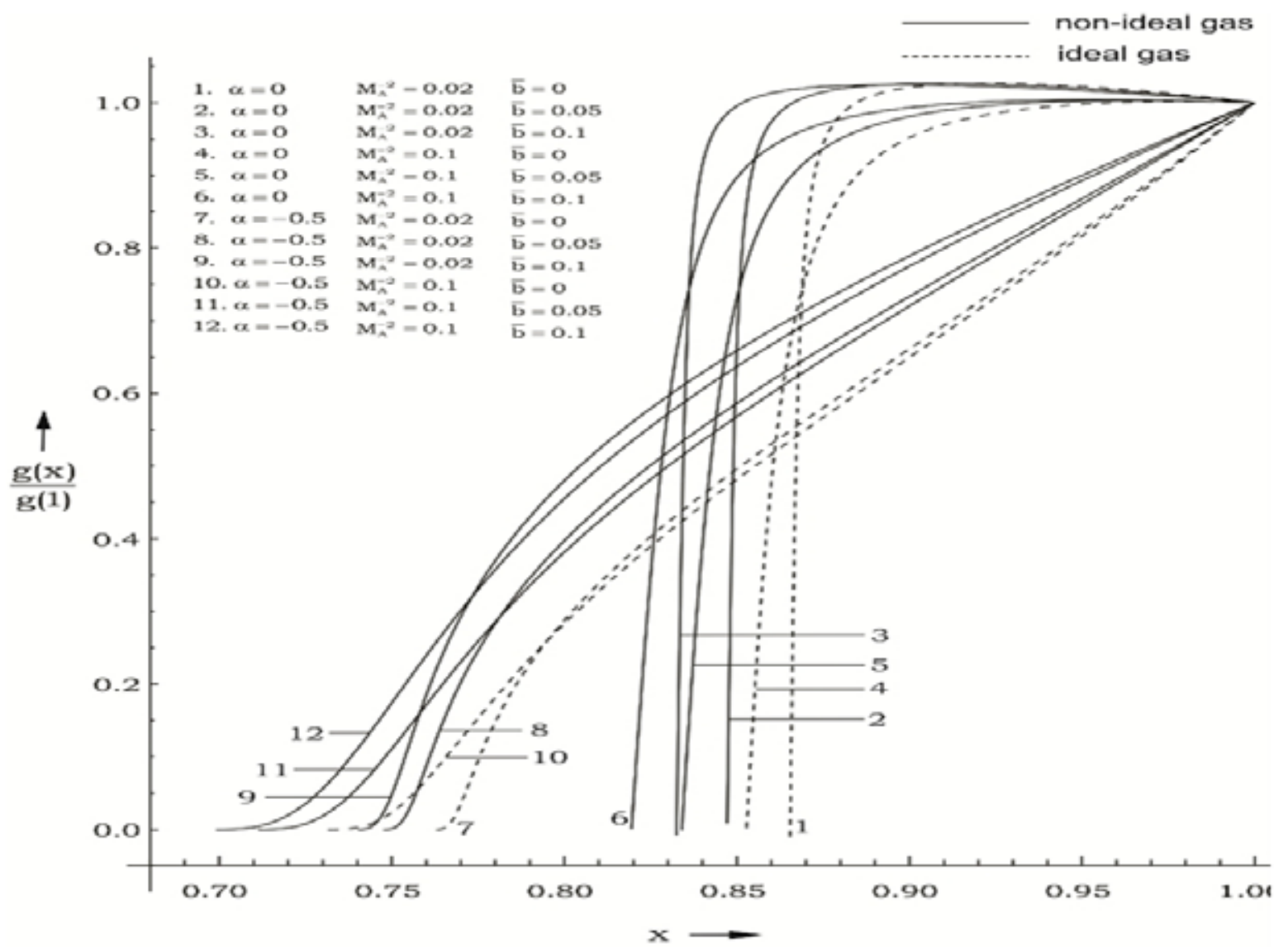

Figure 3. Variation of the reduced density in the flow-field behind the shock front

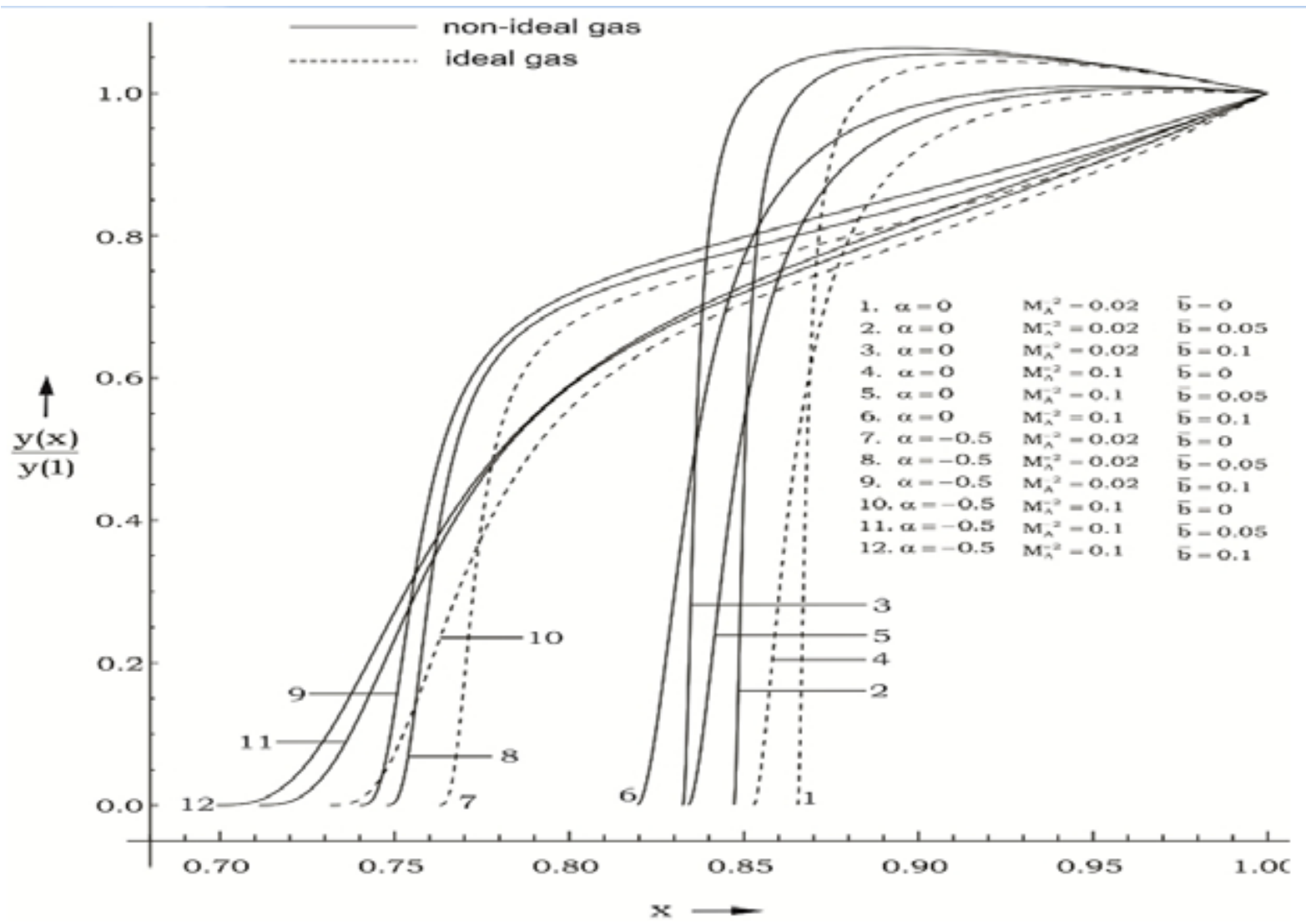

Figure 4. Variation of the reduced pressure in the flow-field behind the shock front 


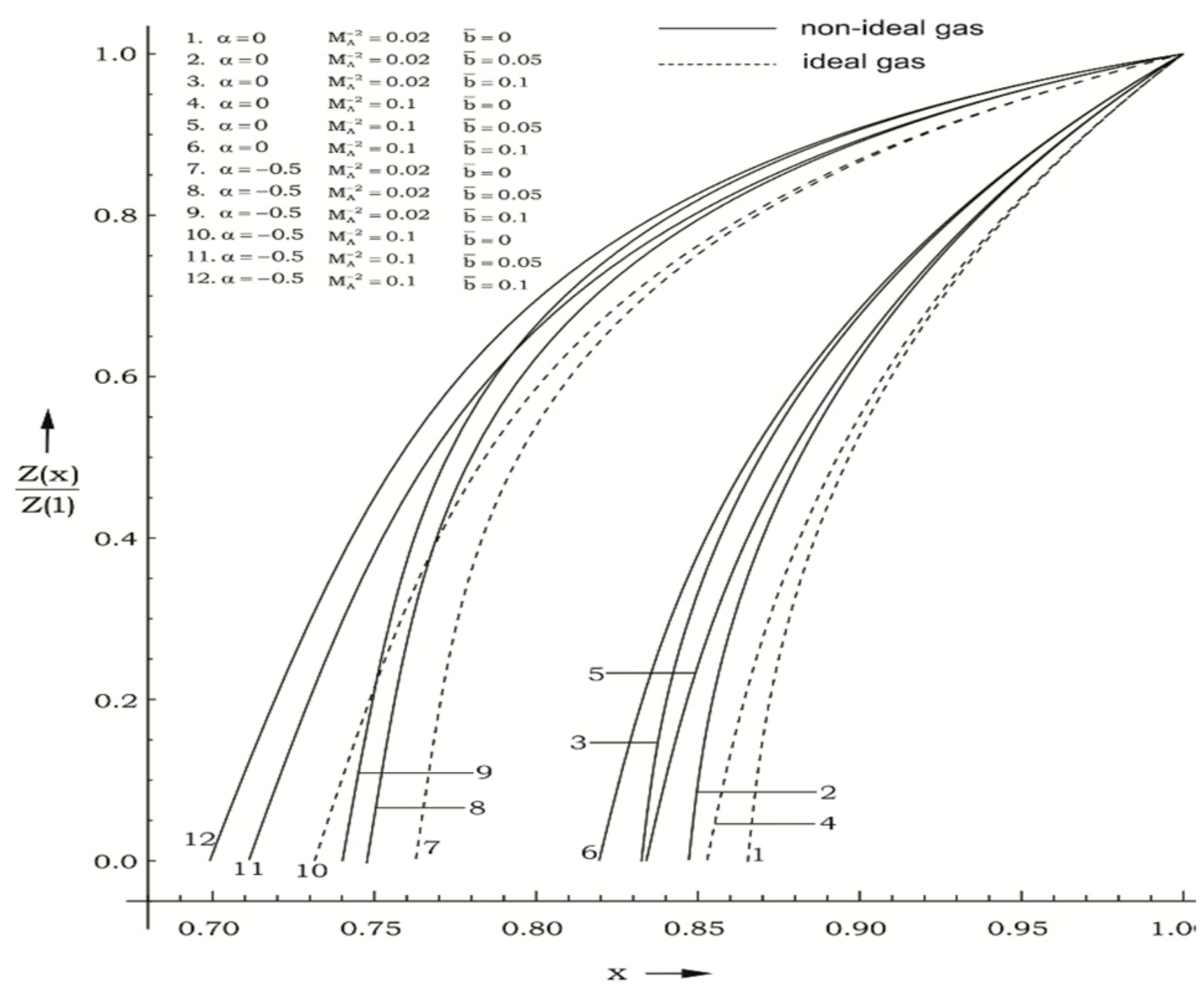

Figure 5. Variation of the reduced azimuthal velocity in the flow-field behind the shock front

Figure 1 shows that the radial velocity $\mathrm{w}$ increases from the shock front to the inner expanding surface when $\alpha=0$; whereas its increase is very slow in almost all the cases of $\alpha=-0.5$. In these cases $(\alpha=-0.5)$, it starts to decrease after attaining a maximum near the inner surface. The nature of the radial velocity profile in the flow-field behind a gas-ionizing shock is different from that behind a magnetogasdynamic shock, where it decreases from shock front to inner surface in the cases of $\alpha=-0.5$ (Vishwakarma et al.[11]).

Figure 2 shows that the magnetic field s increases abruptly near the inner contact surface when the initial magnetic field is weak $\left(\mathrm{M}_{\mathrm{A}}^{-2}=0.02\right)$. This behaviour of the magnetic field is removed in the cases of strong initial magnetic field $\left(\mathrm{M}_{\mathrm{A}}^{-2}=0.1\right)$. The magnetic field does not exhibit the behaviour of abrupt increase near the inner surface in the case of magnetogasdynamic shock (see figure 3 of Vishwakarma et al.[11]).

Figures 3, 4 and 5 show that the density g, pressure y and azimuthal velocity $\mathrm{z}$ decrease from shock front to the inner contact surface. The density and pressure fall abruptly near the inner surface when $\alpha=0$ and $\mathrm{M}_{\mathrm{A}}^{-2}=0.02$. This ten- dency of density and pressure is reduced if $\alpha$ is decreased $(\alpha=-0.5)$ or if $\mathrm{M}_{\mathrm{A}}^{-2}$ is increased $\left(\mathrm{M}_{\mathrm{A}}^{-2}=0.1\right)$.

From tables 1 and 2 and figures 1 to 5 , it is found that the effects of an increase in the value of $\mathrm{M}_{\mathrm{A}}^{-2}$ (i.e. the effects of an increase in the strength of ambient magnetic field) are

(i) to decrease the radial velocity and azimuthal magnetic field at a point in the flow-field behind the shock front, but to increase the azimuthal velocity (see figures 1, 2 and 5);

(ii) to decrease the density and pressure, except in a region near the inner contact surface. The decrease in density is small for $\alpha=-0.5$ in comparison to that for $\alpha=0$ (see figures 3 and 4 );

(iii) to decrease the slopes of the profiles of the density, pressure and azimuthal magnetic field, i.e. to reduce the tendency of abrupt fall of the density and pressure and abrupt increase of the azimuthal magnetic field as we move inwords from the shock front (see figures 2, 3 and 4); and

(iv) to increase the distance of the inner contact surface from the shock front (see table 1), but this increase is small in comparison with that in the case of magnetogasdynamic shock studied by Vishwakarma et al.[11].

Thus the increase in the strength of the magnetic field has 
decaying effect on the ionizing shock wave, but it is less in comparison with that in the case of magnetogasdynamic shock.

The effects of an increase in the value of the parameter of the non-idealness of the gas $\bar{b}$ are

(i) to increase the radial and azimuthal velocities at a point in the flow-field behind the shock but to decrease the azimuthal magnetic field, in general. The increase in the radial velocity is significant in the cases when $\alpha=-0.5$ (see figures 1, 2 and 5);

(ii) to increase the density and pressure, in general (see figures 3 and 4); and

(iii) to increase the distance between inner contact surface and the shock front, and $\beta=\rho_{1} / \rho_{2}$ (see tables 1 and 2).

Therefore the non-idealness of the gas has decaying effect on the ionizing shock wave as in the case of magnetogasdynamic shock.

The effect of an increase in the value of the index for variation of azimuthal magnetic field $\alpha$, i.e. the effects of an increase in the value of the index for variation of the angular velocity of the ambient medium $d$ are

(i) to increase the shock velocity (see equation (3.18));

(ii) to decrease the distance of inner expanding surface from the shock front. It means that the shock is stronger when the ambient magnetic field is uniform $(\alpha=0)$ in comparison with that when it is decreasing $(\alpha=-0.5)$. It also means that the shock is stronger when the angular velocity of the ambient medium is slowly decreasing (see the relations (2.19) and (3.20)); and

(iii) to increase the slopes of profiles of all the flow variables in the flow-field behind the shock. (see figures 1-5).

\section{Conclusions}

In the present paper, similarity solutions are obtained for the flow-field behind a gas-ionizing cylindrical shock wave propagating in a rotating non-ideal gas in presence of an azimuthal magnetic field. On the basis of this study one may draw the following conclusions.

(i) the shock velocity varies as the ambient azimuthal velocity (i.e. as the ambient magnetic field).

(ii) the presence of magnetic field has decaying effect on the ionizing shock wave but it is less in comparison with that on the magnetogasdynamic shock;

(iii) the non-idealness of the gas also has decaying effect on the ionizing shock wave, and it is almost of the same intensity as that on the magnetogasdynamic shock;

(iv) in the case when the initial magnetic field or the initial angular velocity of the medium is decreasing with distance (i.e. when $\alpha=-0.5$ ), the nature of the radial velocity profiles in the flow-field behind the ionizing shock is significantly different from that behind the magnetogasdynamic shock; and

(v) when the initial magnetic field is weak $\left(\mathrm{M}_{\mathrm{A}}^{-2}=0.02\right)$ the magnetic field increases abruptly near the inner contact surface. The magnetic field does not exhibit this behavior in the case of the magnetogasdynamic shock.

\section{REFERENCES}

[1] Sedov L. I.,Similarity and Dimensional Methods in Mechanics. London: Academic Press, 1959.

[2] Roberts P. H. and Wu C. C.,Structure and stability of a spherical implosion. Phys. Lett. A, 1996, 213, 59-64.

[3] Lee T. S. and Chen T.,Hydromagnetic interplanetary shock waves. Planet. Space Sci., 1968, 16, 1483-1502.

[4] Summers D.,An idealized model of a magnetohydrodynamic spherical blast wave applied to a flore produced shock in the solar wind. Astron. Astrophys., 1975, 45, 151-158.

[5] Chaturani P.,Strong cylindrical shocks in a rotating gas. Appl. Sci. Res., 1970, 23, 197-211.

[6] Sakurai A.,Propagation of spherical shock waves in stars. J. Fluid Mech., 1956, 1, 436-453.

[7] Nath O.,Ojha S. N., Takhar H. S.,A study of stellar point explosion in a radiative magneto-hydrodynamic medium. Astrophys. Space Sci., 1991, 183, 135-145.

[8] Ganguly. A. and Jana M.,Propagation of shock wave in a self-gravitating radiativemagnetohydrodynamic non-uniform rotating atmosphere. Bull. Cal. Math. Soc., 1998, 90,77-82.

[9] Roberts P. H. and Wu C. C.,Structure and stability of a spherical implosion. Phys. Lett. A, 1996, 213, 59-64.

[10] Roberts P. H. and Wu C. C., The shock-wave theory of sonoluminescene, in Shock Focussing effect in Medical Science and Sonoluminescene (Eds. R.C. Srivastava, D. Leutloff, K. Takayama, H. Groning ): Springer Verlag, 2003.

[11] Vishwakarma J. P.,Mauraya A. K.,Singh K. K.,Self-similar adiabatic flow headed by a magnetogasdynamics cylindrical shock wave in a rotating non-ideal gas, Geophys. Astrophys. Fluid Dyn.,2007, 101, 155-167.

[12] Greenspan H. P.,Similarity Solution for a cylindrical Shock-magnetic field interaction, Phys. Fluids, 1962, 5, 255-259.

[13] Greifinger C. and Cole J. D.,Similarity Solution for cylindrical Magnetohydrodynamic (magnetogasdynamic) Blast Waves. The Phy. of Fluids,1962, 5, 1597-1607.

[14] Christer A. H.,Self-similar cylindrical ionizing shock and detonation waves, Z. Angew Math. Mech., 1972, 52, 11-22.

[15] Rangarao M. P. and Ramana B. V.,Self-similar cylindrical magnetogasdynamic and ionizing shock waves, Indian Int. J. Engng. Sci., 1973, 11, 337-351.

[16] Singh J. B.,Self-similar cylindrical ionizing shock waves with radiation heat flux, Indian. J. Tech., 1983, 21, 315-318.

[17] Whitham G. B.,On the propagation of shock waves through regions of non-uniform area or flow. J. Fluid Mech., 1958, 4, 337-360.

[18] Singh J. B. and SrivastavaS. K.,Cylindrical ionizing shock waves in a non-ideal gas, J. Purv. Acad. Sci. Jaunpur, 1991, 2, 
39-46.

[19] Vishwakarma J. P. and Pandey S. N.,Converging cylindrical shock waves in a non-ideal gas with an axial magnetic field, Def. Sci. J., 2006, 56, 721-731.

[20] Rogers M. H.,Similarity flows behind strong shock waves. Quart. J. Mech. Appl. Math., 1958, XI (II), 411-422.

[21] Freeman R. A., Variable energy blast wave. J. Phys. D., 1968, $1,1697-1710$.

[22] Director M. N. and Dabora E. K.,An experimental investigation of variable energy blast waves. Acta. Astronaut.,1977, 4, 391-407.

[23] Freeman R. A. and Craggs J. D.,Shock waves from spark discharges. J. Phys. D. Appl. Phys., 1969, 2, 421-427.

[24] Rosenau P.,Equatorial propagation of axisymmetric magnetohydrodynamic shocks. II. Phys. Fluids., 1977, 20, 1097-1103.

[25] Rosenau P. and Frankenthal S., Shock disturbances in a thermally conducting solar wind. Astrophys. J., 1976, 208, 633-637.

[26] Vishwakarma J. P. and Yadav A. K.,Self-similar analytical solutions for blast waves in homogeneous atmospheres with frozen-in-magnetic field. Eur. Phys. J. B., 2003, 34, 247-253.

[27] Vishwakarma J.P. and Singh Mahendra, Self-similar cylindrical ionizing shock waves in a non-ideal gas with radiation heat-flux. Applied Mathematics, 2012, 2, 1-7. 